\title{
Chemical Structure Role of Cell-Membrane Hydrocarbon Chain in Interactions with Antihypertensive Drugs (SARTANs)
}

\author{
A. HODZIC $^{1}$, T. MAVROMOUSTAKOS ${ }^{2}$, P. ZOUMPOULAKIS ${ }^{2}$, \\ M. RAPPOLT ${ }^{3}$, P. LAGGNER ${ }^{3}$, G. PABST $^{3}$ \\ ${ }^{1}$ Research Center Pharmaceutical Engineering GmbH, Graz, Austria \\ ${ }^{2}$ National Hellenic Research Foundation, Institute of Organic and Pharmaceutical Chemistry, Athens, \\ Greece \\ ${ }^{3}$ Austrian Academy of Sciences, Institute of Biophysics and Nanosystems Research, Graz, Austria, \\ E-mail: aden.hodzic@tugraz.at (A. Hodzic)
}

Sci Pharm. 2010; 78: 697

doi:10.3797/scipharm.cespt.8.PPAT08

Stressful life style of modern human societies leads to many diseases and one of the growing indispositions related to this fact is hypertension. Thus, there is a strong need for novel effective drugs. One of the new categories of antihypertensive drugs consists of $\mathrm{AT}_{1}$ antagonists (SARTANs), whose action is based on blocking of the active site of the $\mathrm{AT}_{1}$ receptor. The aim of the present study is to contribute to a basic understanding of the their molecular mode of action on membranes. We studied influences on the binary mixtures of DMPC/cholesterol and POPC/cholesterol. Losartan led to a complete loss of positional correlations between adjacent bilayers for all single component model membranes. This can be explained by the negative surface charged conferred to the bilayers upon losartan insertion. The effect was however, counterbalanced upon the addition of cholesterol. Both, POPC and DMPC bilayers exhibited no positional correlations up to $5 \mathrm{~mol} \%$ cholesterol, respectively. However, only POPC remained uncorrelated at $20 \mathrm{~mol} \%$ and above, while DMPC/cholesterol bilayers exhibited multilamellar vesicles, i. e. a reentrant transition of the positionally uncorrelated bilayers into correlated ones. Our results may be understood in view of the different hydrocarbon chain packing densities in saturated versus unsaturated bilayers and their respective affinities to interaction with cholesterol. This shows that the insertion of losartan into the membrane may be overridden by a tighter bilayer interface and emphasizes the role of hydrocarbon chain composition in its mode of action. Additionally, we also the influence of the SARTANs (e.g. losartan, candesartan-cilexiril and valsartan) on the global structure of phospholipid bilayers composed of pure dimyristoyl phosphatidylcholine (DMPC), palmitoyl oleoyl phosphatidylcholine (POPC) and dipalmitoil phosphatidylcholine (DPPC) applying synchrotron smallangle $x$-ray scattering. Valsartan is the only one which shows a disruption of the neutral DPPC bilayer, more visible at higher drug concentrations. This could be attributed to the valsartan chemical structure, which poses a carbonyl acid (carboxyl) group providing higher polarity compared to the other two drugs in the neutral environment. 